\title{
Comparative Study of the Temperature of the Masseter and Temporal Muscles of Patients with and Without Temporomandibular Dysfunctions Through Thermography
}

\author{
Isadora Vasconcelos Machado de Brito ${ }^{1}$, João Paulo Alves do Couto ${ }^{2, *}$, Iasmin Fonseca Bertoldi ${ }^{1}$, Pri- \\ sicla Silva Fadini ${ }^{3}$ \\ ${ }^{1}$ Integrated College of Southern Bahia, Brazil. \\ ${ }^{2}$ Department of physiotherapy, College of Southern Bahia, Brazil. \\ ${ }^{3}$ Department of Medicine, College of Southern Bahia, Brazil.
}

How to cite this paper: Isadora Vasconcelos Machado de Brito, João Paulo Alves do Couto, Iasmin Fonseca Bertoldi, Prisicla Silva Fadini. (2021) Comparative Study of the Temperature of the Masseter and Temporal Muscles of Patients with and Without Temporomandibular Dysfunctions Through Thermography. International Journal of Clinical and Experimental Medicine Research, 5(3), 236-242. DOI: $10.26855 /$ ijcemr.2021.07.001

Received: March 22, 2021

Accepted: April 20, 2021

Published: May 8, 2021

*Corresponding author: João Paulo Alves do Couto, Department of physiotherapy, College of Southern Bahia, Brazil.

Email: jcouto@unece.br

\begin{abstract}
Temporomandibular dysfunctions (TMD) consist of a collection of clinical signs and symptoms involving the temporomandibular joint and the muscles of chewing, with pain being the most constant symptom. Infrared thermography is a noninvasive, painless, and non-radioactive analytical instrument capable of examining physiological functions under body temperature control. Objective: To compare the temperature of the masseter and temporal muscles of people with and without temporomandibular dysfunctions. Methodology: This is an experimental cross-sectional field research, in which 25 individuals were selected, with ages ranging between 18 and 40 years and applied the anamnetic self-application questionnaire of Silveira et al. where the presence and severity of TMD was determined and then the temperature of the masseter and temporal muscle was analyzed through thermography. Results and Discussion: They indicate that there is a statistical difference of temperature between individuals without TMD in relation to individuals with severe TMD. There was no significant statistical difference between individuals with mild and moderate TMD compared to those without TMD. Conclusion: Therefore, even though there are not many studies regarding the use of infrared thermography in temporomandibular dysfunctions, the results of this study were positive regarding the use of thermography to detect the increase of temperature of the masseter and temporal muscles in individuals with severe TMD when compared to healthy individuals.
\end{abstract}

\section{Keywords}

Temporomandibular Disorders, Thermography, Masseter, Temporal

\section{Introduction}

The temporomandibular joints (TMJ) are two of the most incessantly used joints of the body, since they move about 1,500 to 2,000 times a day, besides being responsible for important functions such as speaking, yawning, chewing, swallowing, breathing, kissing or sucking [1].

This joint is considered as one of the most complex in the human body. And it is an arthroidal slang joint for 
making hinge (ginglimoidal) and slip (artroidal) movements [2].

Temporomandibular dysfunctions (TMD) consist of a collection of clinical signs and symptoms involving the temporomandibular joint and the muscles of mastication, with pain being the most constant symptom [3]. Its etiology is multifactorial and may be associated with traumatic or degenerative lesions of TMJ, occlusion alteration, skeletal problems, internal disc disorder, hypomobility or joint hypermobility, joint or muscular dysfunction, in addition to psychological factors and deleterious habits, being more prevalent in women, with a ratio of 4:1 men [1].

Infrared thermography is a noninvasive, painless, and non-radioactive analytical instrument capable of examining physiological functions under body temperature control. For cases of temporomandibular dysfunctions, thermography can assist in the diagnosis and evaluation because it is an exam capable of detecting red light and the temperature emitted by the body, showing anatomical abnormalities and existing physiological changes $[4,5]$.

The infrared image (IR) is capable of detecting a temperature difference of $0.1^{\circ} \mathrm{C}$ between two points of $1 \mathrm{~mm}^{2}$ distance, while our hand is only able to perceive thermal differences from $2^{\circ} \mathrm{C}$. In addition, our thermal perception is not very accurate [6]. In view of this, the use of thermography will enable the more accurate verification of temperature changes that the musculature may suffer from temporomandibular dysfunctions.

\section{Materials and methods}

\subsection{Study nature}

This is an experimental, cross-sectional field research.

\subsection{Field and study setting}

The survey was conducted in the city of Eunápolis, which is in the extreme south of Bahia, with an average of one hundred and twelve thousand three hundred and eighteen inhabitants (112, 318). Capital is generated by trade.

The research was carried out on the campus of the Faculdades Integradas do Extremo Sul da Bahia (Unesulbahia), located on Highway 367, Km 14, Eunápolis. The laboratory is approximately $78 \mathrm{~m}^{2}$ and was air-conditioned at $21^{\circ} \mathrm{C}$.

\subsection{Study population}

This is a study with men and women aged 18-40 years (this age group was chosen because there was no difference in the prevalence of TMD), academics of business courses, accounting sciences, law, nursing and physiotherapy at Faculdades Integradas do Extremo Sul da Bahia. Which were chosen randomly and allocated into four groups. After the application of the anamnetic questionnaire, Silveira et al. (2007), the individuals were placed in the following groups: without TMD, with mild TMD, moderate TMD and with severe TMD. The study consisted of 30 individuals, but after applying the inclusion and exclusion criteria, 25 individuals were selected.

The inclusion criteria were: the term of free and informed consent, answer the questionnaire applied, be aged between 18-40 years. The exclusion criteria were: not answering the questionnaire applied, being less than 18 years old or more than 40 years old, having a beard, having a history of depression or anxiety and having suffered some trauma or surgery in the orofacial region recently.

\subsection{Procedures}

Previous visits were carried out where the first contact was with the director or the pro-director of Unesulbahia for the authorization of the data collection, in the second moment was passing through all the rooms to invite and explain the students interested in the research. Then, the signatures of the informed consent form were collected and the questionnaire was answered. Finally, thermographic images of the masseter and temporal muscles of the volunteers with and without temporomandibular dysfunctions were made.

\subsection{Instructions for data collection}

For data collection, we used the validated questionnaire, anamnetic self-application by Silveira et al. (2007), which aims at the detection of TMD. The questionnaire consists of 10 objective questions, in which the answer options are: yes, sometimes and no. Where the yes is worth 2 points, the sometimes worth 1 point and the not worth 0 . According to Couto et al. [7], after the sum of the 10 questions, the following values of score were stipulated: 0 to 3 refers to people who do not have TMD; from 4 to 8 carriers of mild TMD; 9 to 14 moderate TMJD and 15 to 22 severe TMJD.

In the thermographic examination, the infrared camera was positioned at the height of the volunteers' eyes, at a 
distance that the entire area of the bust and face is within the perimeter of the camera, with the largest point of the body's infrared image occupying the entire monitor.

The thermographic images were taken on the right and left sides of the volunteers. The temperature analysis box was placed from the medium to the temporal insertion and along the origin and insertion of the masseter. The maximum temperature of $38^{\circ} \mathrm{C}$ and a minimum of $27^{\circ} \mathrm{C}$ were used for image standardization (Figure 1).

Some instructions were given beforehand. On the day prior to the examination, individuals were instructed not to wax the area that will be used, not to exercise, physical therapy, acupuncture or strenuous physical activities; to avoid the consumption of coffee, black tea and alcoholic beverages (due to the vasodilator effect).

Prescriptions on the day of the exam were not to smoke 2 hours before; avoid applying lotions or face creams 1 hour before and avoid anti-inflammatory, analgesic and decongestant medications.

At the time of the examination, the guidelines were to maintain the air-conditioned environment with the temperature at $21^{\circ} \mathrm{C}$, measured through the thermo-hygrometer for the cutaneous thermal stabilization; the men were shirtless and the women "hopefully falling" or cotton bra (so as not to affect skin temperature visualization); metallic objects, earrings, necklaces, piercing, etc. were removed (Figure 2).

The infrared camera that was used in the study is the FLIR ONE PRO (Figure 3). It has the ability to measure temperatures between $-20^{\circ}$ and $400^{\circ} \mathrm{C}$, with thermal resolution of 19,200 pixels and image resolution of $1440 \times 1080$.

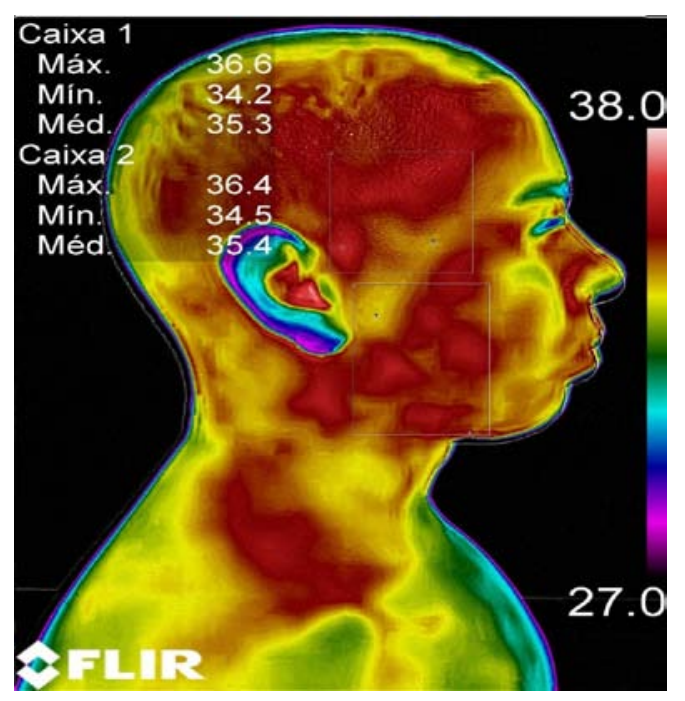

Figure 1. Thermographic image of the right masseter and temporal muscles.

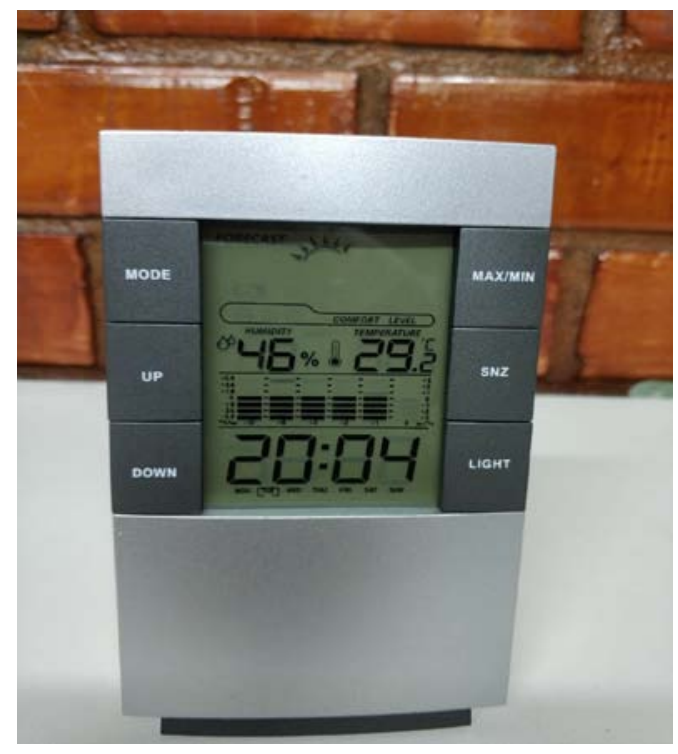

Figure 2. Thermohygrometer. 


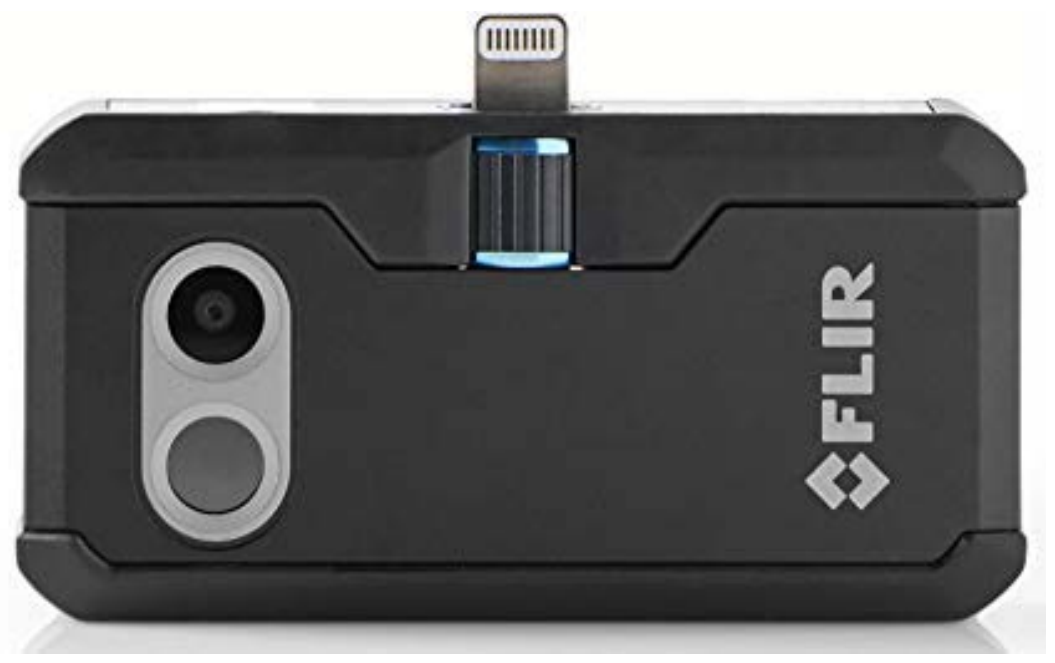

Figure 3. Infrared Thermal Imager.

Source: https://www.amazon.com/FLIR-ONE-Thermal-Imaging-Camera/dp/B07DM8DG1B.

\subsection{Data analysis}

For the statistical analysis, Bioestat 5.0 was used. After the descriptive analysis of the data, the Shapiro-Wilk test was applied to verify the normality of data distribution. The variables were nonparametric. Therefore, the Wilcoxon test was used to compare the variables between the evaluations of both groups. The level of significance adopted was $\mathrm{P}=0.05$ for all analyses. The Sigma Plot program was used to make the graphs.

\subsection{Ethical aspects}

This study was developed in accordance with Resolution 466/12 of the National Health Council, which regulates research with human beings, considering the observance of beneficence, non-maleficence, absence of risks and damages, with guarantee of anonymity to the study subjects. The data collection of the research was only started after the approval of a research ethics committee and the data collection took place after the acceptance of the study subject and the signing of the informed consent form.

\section{Results}

The study consisted of 25 volunteers (19 women and 6 men), aged between 20-40 years. Two images were taken of each volunteer, being in lateral projections right and left.

During the analysis of the left temporal muscle temperature data, the results indicate that there is a significant statistical difference $(\mathrm{p}=0.02)$ between individuals who do not have TMD in relation to individuals who have severe TMD (Figure 4). When comparing the right and left temporal muscle temperature of the volunteers with mild and moderate TMD compared to those without TMD, the results suggest that there is no significant statistical difference.

Despite having a difference in the graph, the results did not indicate significant statistical differences in the right temporal muscle temperature of individuals without TMD in relation to individuals with severe TMD.

In the course of the analysis of the right masseter muscle temperature data, the statistical results suggest significant differences ( $\mathrm{p}=0.008$ ) between individuals without TMD and individuals with severe TMD. On the left side of the masseter, the statistical data also indicate significant changes in temperature $(\mathrm{p}=0.02)$ among volunteers without TMD and volunteers with severe TMD (Figure 5).

\section{Discussion}

The results indicate that there is a significant statistical difference in temperature between individuals without TMD in relation to individuals with severe TMD. There was no significant statistical difference between individuals with mild and moderate TMD compared to those without TMD. Dibai Filho et al. [8], conducted a similar study, but only with women, where the results obtained agree in part with this study, where they indicate that there is no significant statistical difference between individuals with mild and moderate TMD compared to those without TMD, however, no difference was observed in patients with severe TMJD. 


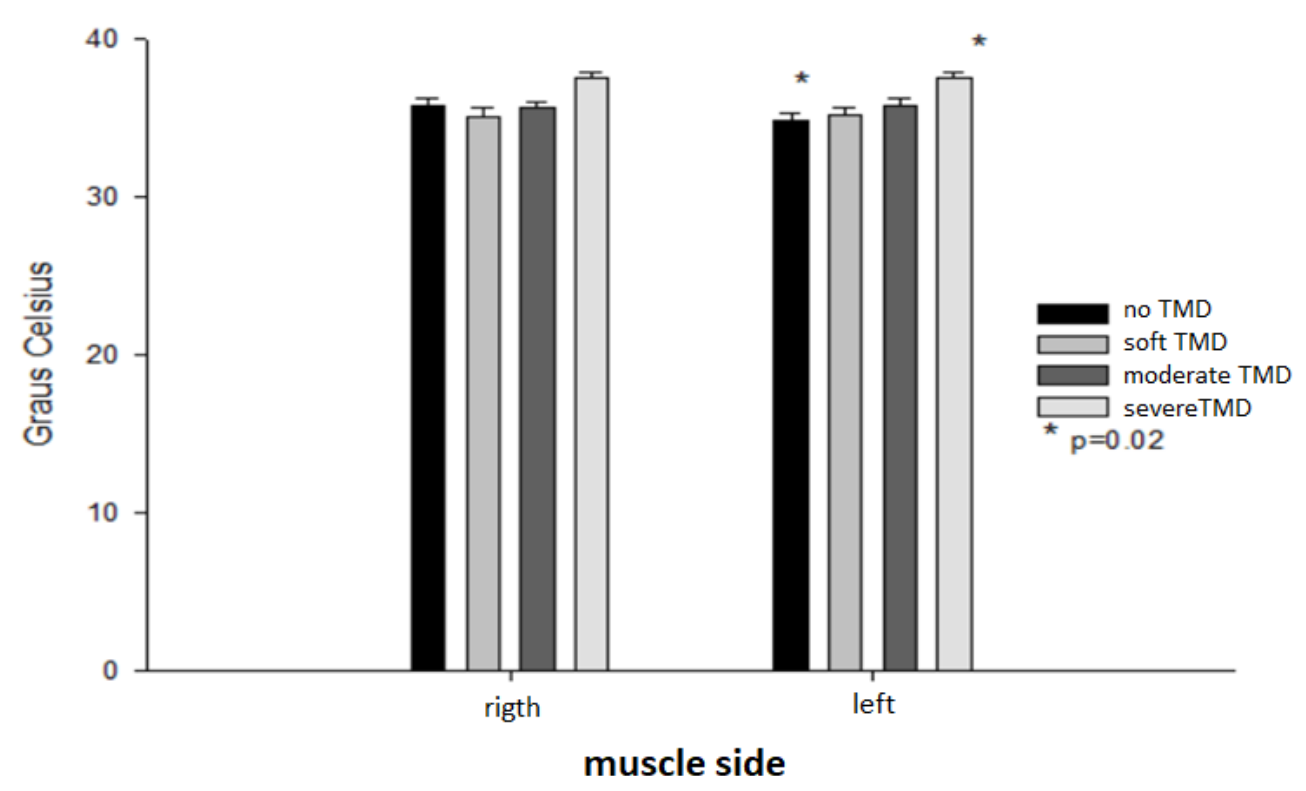

Figure 4. Analysis of the maximum temperature of the right and left temporal of the volunteers.

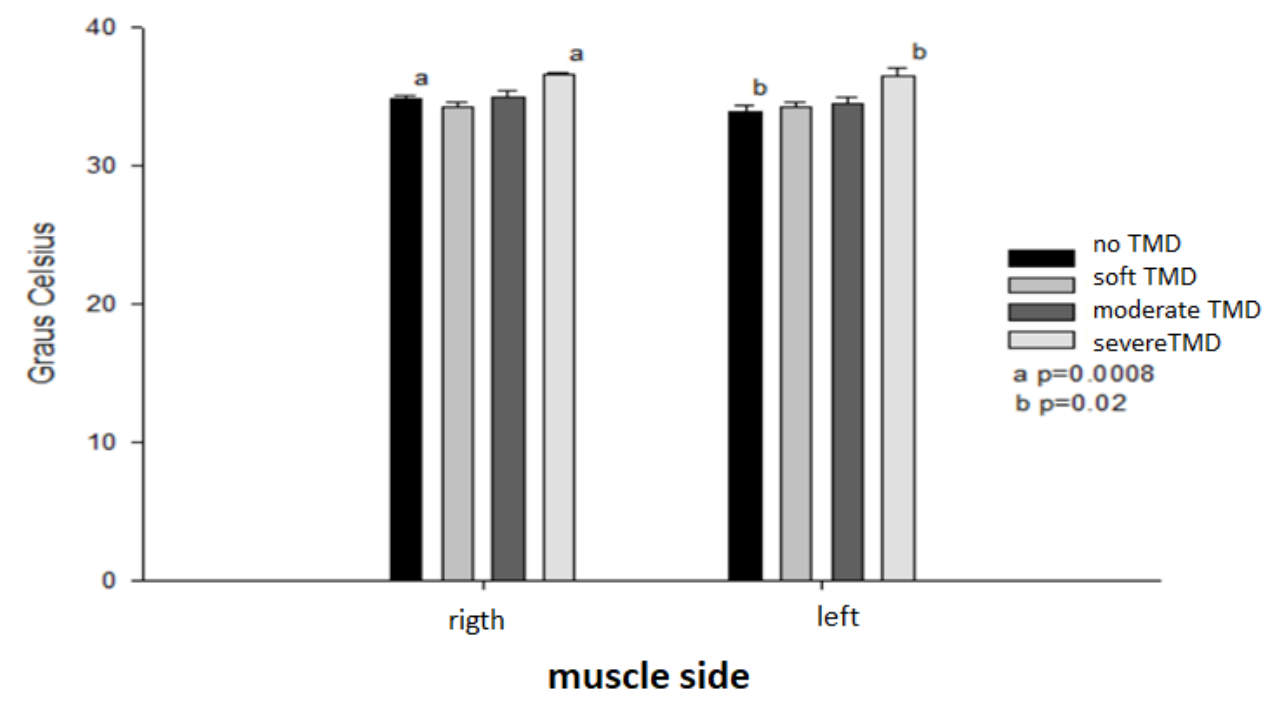

Figure 5. Analysis of the maximum temperature of the right and left masseter of the volunteers.

In studies conducted by the same author in 2014 and 2015, positive results were obtained. Dibai Filho et al. [9] used 30 women with a diagnosis of TMD and analyzed the masseter and temporal muscles, the results indicated a significant increase in the temperature of the temporal muscle; however, no significant changes were noticed in the masseter muscle. Dibai Filho [10], observed an increase in temperature throughout the right and left TMJ region of the volunteers with severe TMD when compared to those without TMD. In addition, no significant difference was observed in the comparison of those with mild and moderate TMD in relation to those without TMD.

In agreement, studies conducted by Clemente [11] and Schadeck [12] also obtained positive results in the analysis, resulting in increased temperature of the masseter and temporal muscles in patients with TMD, despite the study by Schadeck [12] have been performed with a low number of volunteers and both have not subdivided the carriers of TMJD as to the degree of dysfunction.

On the other hand, the authors Rodrigues-Bigaton et al. [13] and Haddad et al. [14], contradict the above results, because the results demonstrated by both of them show a reduction in muscle temperature in TMD carriers in comparison to healthy ones. However, the authors only used women in the study, besides Rodrigues-Bigaton et al. [13] used a longer time of climatization in the volunteers, from 20 to 22 minutes, not respecting the thermoregulation suggested by the literature that proposes a shorter time between 8 and 16 minutes. Although Haddad et al. [14] res- 
pected the thermoregulation suggested by the literature, the study samples suggested a reduction in the temperature of the masseter and temporal muscles of the volunteers with TMD in relation to the control group.

In the bibliographic reviews conducted by Presídio, Wanderley e Medrado [15] and Schnorr [16], where the objective was to observe the efficiency of the use of thermography in the diagnosis of temporomandibular dysfunctions, the results were positive. Therefore, both authors suggest the use of thermographic examination both for the diagnosis and for the evaluation of TMD carriers.

The research conducted by Barbosa [17] suggests that patients with TMD have a temperature similar to those who do not have TMD. However, during the analysis of the present study, no significant temperature changes were observed when comparing all TMD carriers to those without TMD. The significant differences in temperature were only noticed when TMD carriers were subdivided into groups, being observed only in the comparison of individuals with severe TMD compared to those without TMD.

The results obtained in the research conducted by Costa et al. [18] recommend the use of thermography as an aid tool in the evaluation of TMD. Although they were not mentioned in which muscles the increase in temperature was observed and the study used only women as volunteers. However, Woúniak et al. [19], says that thermography is not indicated as a single method for the diagnosis of TMD, since it alone is not as effective in the evaluation of TMD carriers, but when associated with the masticatory test it presents positive results.

According to Lucas and Briochi [5], thermal differences of up to $0.24^{\circ} \mathrm{C}$ are considered within the normality standard, and that differences from $0.3^{\circ} \mathrm{C}$ represent indications of mild abnormality, $0.6^{\circ} \mathrm{C}$ suggests moderate abnormalities and $1^{\circ} \mathrm{C}$ significant abnormality. Because they were minimal differences in temperature between normal, mild and moderate, they could have gone unnoticed in the present study, justifying the fact that no significant differences were perceived between these groups.

\section{Conclusion}

Temporomandibular dysfunctions have diversified symptomatology and multifactorial etiology, which can hinder the accuracy of the diagnosis, making it necessary several forms of tests for accuracy.

Thermography has been shown to be an excellent instrument for body temperature analysis, as it is easy to apply and does not present any risk to the patient, and can be applied many times. Although some authors disagree on the effectiveness of thermography for the diagnosis of TMD, the vast majority of current studies recommend its use.

Therefore, even though there are not many studies regarding the use of infrared thermography in temporomandibular dysfunctions, the results of this study showed are positive as to the use of thermography to detect the increased temperature of the masseter and temporal muscles in individuals with severe TMD when compared to healthy individuals.

\section{References}

[1] Garcia, J. D. and Oliveira, A. A. D. C. (2011). Physiotherapy in the signs and symptoms of temporomandibular joint (TMJ) dysfunction. Hórus Magazine, Ourinhos, 2011 Jan, v. 1, n. 6, p.111-122.

[2] Magee, D. J. (2010). Musculoskeletal Assessment. 5th ed. Barueri: Manole, 2010, p. 1223.

[3] Martins, R. J., et al. (2010). Stress levels and sleep quality in patients with temporomandibular joint dysfunction. Science and Dentistry Magazine, 2010 Jan, v. 1, n. 25, pp. 32-36.

[4] Côrte, A. C. R. and Hernandez, A. J. (2016). Infrared medical thermography applied to sports medicine. Brazilian Journal of Sports Medicine, 2016 mai, pp. 315-319.

[5] Lucas, R. W. C. and Brioschi, M. L. (2016). Thermography applied to physiotherapy. Florianópolis: Sistema Wallace Consultoria Ltda, 2016. P. 125.

[6] Brioschi, M. L., Teixeira, M. L, Yeng, L.T., and Silva, F. M. R. M. (2012). Medical Thermography Manual (Based on the International Consensus and Guidelines for Medical Thermography). 2012, São Paulo: Andreoli.

[7] Couto, J. P. A., et al. (2011). Prevalence of Temporomandibular Disorders due to Postural Influence in Students of the Physiotherapy Course at UNESULBAHIA. Fisiobrasil, 2011 out, v. 104, n. 15, pp. 16-20.

[8] Dibai-Filho, A. V., de Souza Costa, A. C., Packer, A. C., Rodrigues-Bigaton, D. (2013). Correlation between skin surface temperature over masticatory muscles and pain intensity in women with myogenous temporomandibular disorder. Journal of Back and Musculoskeletal Rehabilitation, Piracicaba, SP, v. 26, n. 3, pp. 323-328, 25 Jul. 2013.

[9] Dibai-Filho, A. V., Packer, A. C., de Souza Costa, A. C., Rodrigues-Bigaton, D. (2014). The chronicity of myogenous temporomandibular disorder changes the skin temperature over the anterior temporalis muscle. Journal of Bodywork and Movement Therapies, Ribeirão Preto, v. 18, n. 3, pp. 430-434, Jul. 2014. Elsevier BV. 
[10] Dibai-Filho, A. V., de Souza Costa, A. C., Packer, A. C., de Castro, E. M., Rodrigues-Bigaton, D. (2015). Women with more severe degrees of temporomandibular disorder exhibit an increase in temperature over the temporomandibular joint. The Saudi Dental Journal. Piracicaba, Sp, pp. 44-49. Jan. 2015.

[11] Clemente, M. P., Faria, C., Coutinho, F. A., Mendes, J., Pinto, J. C., and Amarante, J. M. (2019). The Application of Infrared Thermography as a Quantitative Sensory Measure of the DC/TMD. In: TAVARES, João Manuel R. S.; JORGE, Renato Manuel Natal. VipIMAGE 2019: Proceedings of the VII ECCOMAS Thematic Conference on Computational Vision and Medical Image Processing, October 16-18, 2019, Porto, Portugal. Porto, Por: Springer, 2019, pp. 330-340.

[12] Schadeck, C. A. (2017). Thermography technology applied to mandibular disorders. Management, Technology and Innovation: Electronic magazine of engineering courses, Curitiba, v. 1, n. 1, pp. 50-57, abr. 2017.

[13] Rodrigues-Bigaton, D., Dibai-Filho, A. V., Packer, A. C., de Souza Costa, A. C., de Castro, E. M. (2014). Accuracy of two forms of infrared image analysis of the masticatory muscles in the diagnosis of myogenous temporomandibular disorder. Journal of Bodywork \& Movement Therapies. Piracicaba, Sp, pp. 49-55. Jan. 2014.

[14] Haddad, D. S., Brioschi, M. L., Vardasca, R., Weber, M., Crosato, E. M., Arita, E. S. (2014). Thermographic characterization of masticatory muscle regions in volunteers with and without myogenous temporomandibular disorder: preliminary results. Dentomaxillofac Radiol. 2014; 43: 20130440.

[15] Presidio, L. R., Wanderley, F. G. C., Medrado, A. P. (2016). The use of infrared thermography in dentistry and its specialties: a systematic review. Revista Bahiana de Odontologia, Salvador, 2016, v. 7, n. 2, pp. 155-165, 6 Jul. http://dx.doi.org/10.17267/2238-2720revbahianaodonto.v7i2.960.

[16] Schnorr, K. C. (2017). Comparison of facial thermography and masticatory efficiency of patients with TMD associated with painful symptoms and healthy patients: literature review. 2017. 34 f. Monograph (Specialization) - Dentistry Course, Federal University of Paraná, Curitiba, 2017.

[17] Barbosa, J. S. (2018). Thermographic Analysis of patients with temporomandibular dysfunction. 2018. 90 f. Dissertation (Master) - Dentistry Course, State University of Paraíba, Campina Grande, 2018.

[18] Costa, A. C. S., et al. (2013). Intra and inter-rater reliability of infrared image analysis of masticatory and upper trapezius muscles in women with and without temporomandibular disorder. Brazilian Journal of Physical Therapy. Piracicaba, Sp, pp. $24-31$. fev. 2013.

[19] Woźniak, K., Szyszka-Sommerfeld, L., Trybek, G., Piątkowska, D. (2015). Assessment of the Sensitivity, Specificity, and Accuracy of Thermography in Identifying Patients with TMD. Med Sci Monit, Polônia, pp. 1485-1493, maio 2015. 